\title{
Procedimientas para la supresión del empleo de cimbras en puentes de hormigón armado o sin anman
}

\begin{abstract}
PAUL DUPONT, ingeniero
83610

\section{sinopsis}

En este trabajo se describen detalladamente los distintos medios y procedimientos que pueden emplearse para suprimir la presencia de la cimbra auxiliar, en la construcción de arcos y bóvedas de puentes de hormigón armado o sin armar.

La cimbra, cara y engorrosa, podría evitarse no ya en luces de pequeña importancia con apoyos sobre tierra firme, sino aun tratán. dose de grandes luces y sobre el mar, es decir, sobre estrechos estuarios de grandes ríos, caso en que se halla, por ejemplo, el «Pas-de-Calais».

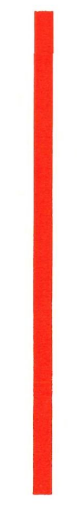
Las cimbras son construccio-
nes difíciles... Los gastos de cimbras en las construcciones cimbras en las construcciones
gravan pesadamente el precio gravan pesadam
de las obras.

J. R. ROBINSON (*)

\section{Métodos clásicos de comstrucción de curcos y bóvedicus de thommigón anmedo}

Inconvenientes de las cimbras

En las primeras aplicaciones del hormigón armado a los arcos y bóvedas de puentes — de finales del siglo XIX-, los constructores se han limitado a utilizar los métodos, en aquel momento en uso, para las obras de mampostería ordinaria, es decir: ejecución con dovelas, por roscas o por anillos, o también por combinación de estos métodos, de donde se deriva el empleo ya clásico, de las cimbras en la construcción de arcos y bóvedas para puentes de hormigón armado.
\end{abstract}

Pero el empleo de cimbras, procedimiento simple y rutinario, acarrea serios inconvenientes; en efecto, las cimbras, algunas veces muy importantes y de ejecución difícil, resultan siempre onerosas y necesitan de mano de obra especializada, bastante escasa en el momento actual. Los períodos de tiempo indispensables, tanto para su colocación como para su desmontaje, aumentan sensiblemente el plazo de tiempo de la ejecución. Por otra parte, las deformaciones a que se hallan sometidas imponen una puesta en carga rigurosamente estudiada. En fin, las molestias que ocasionan constituyen un serio inconveniente para el tráfico en las vías que las atraviesan y, algunas veces, principalmente en el caso de grandes avenidas, un peligro real.

(*) «Piles, Culées et Cintres des Ponts», por J. R. Robinson, Profesor de la Escuela Nacional de Caminos y Puentes (Dunod, Editor, París). 
Además, cuando las cimbras se utilizan para la construcción de arcos o de bóvedas de hormigón armado puede añadirse:

a) que en la ejecución por dovelas aumentan las dificultades de ejecución de encofrados (paso de las capas de armaduras longitudinales a través de encofrados transversales);

b) en el caso de ejecución por roscas se ponen de manifiesto los esfuerzos secundarios como consecuencia de las diferencias de retracción del hormigón entre roscas sucesivas;

c) en el caso de arcos tipo cajón se hacen sentir las dificultades de ejecución según la inclinación variable de los encofrados, de la colocación de armaduras y del hormigonado fraccionado de losas, forjados y alvéolos.

El empleo de cimbras para Io construceión de areos de puentes de hormigón armado, Constituge und "herejúa" técomicu gravosa

Es evidente que en la construcción de arcos de puentes de mampostería ordinaria, en los que la fragilidad exclusiva de todo desplazamiento en período de ejecución impone su construcción directa en la posición definitiva, el empleo ancestral de cimbras costosas continúa siendo una necesidad absoluta.

Contrariamente, en los arcos de hormigón armado, que son infinitamente más resistentes, el empleo de cimbras, procedimiento simple y rutinario, no tiene justificación. Constituye, pues, una "herejía» técnica, puesto que se desconocen a la vez las propiedades esenciales de los arcos de hormigón armado y las ventajas importantes que los constructores pueden obtener, tanto en lo que se refiere a la ejecución de dichos arcos como a la reducción de dichos gastos.

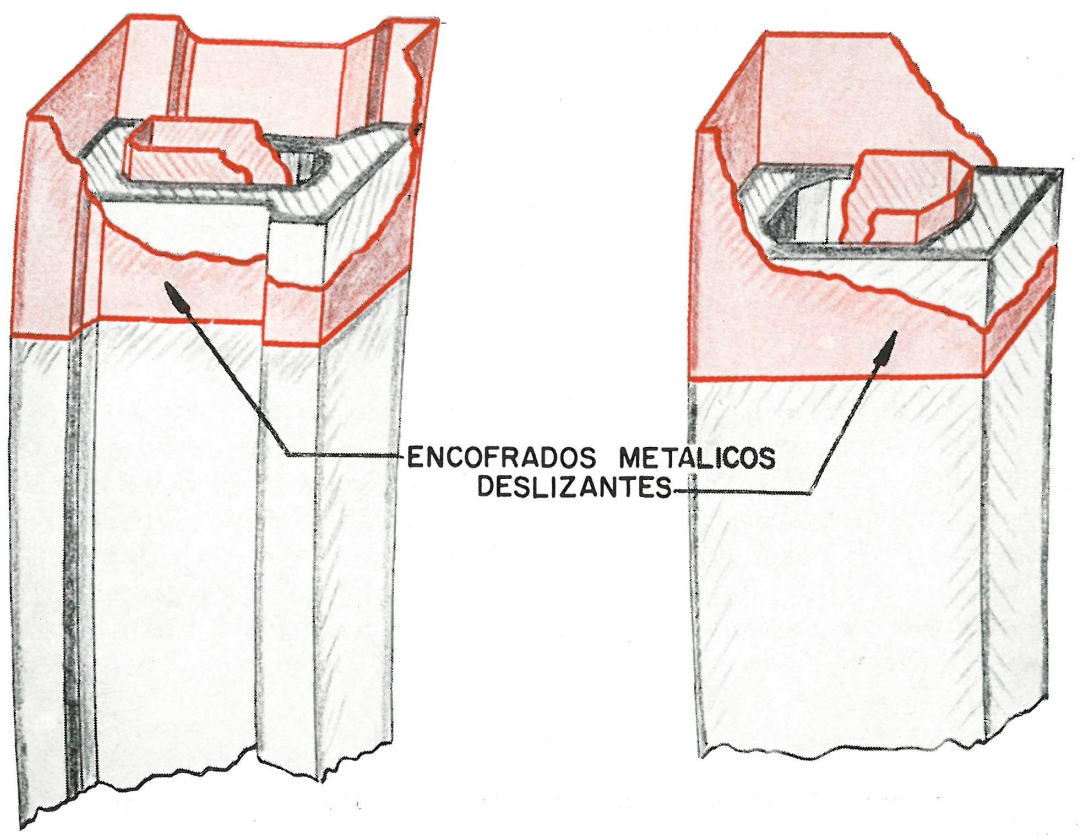


En efecto, y como veremos, el carácter monolítico de los arcos de hormigón armado y su notable rigidez, que hacen posible su construcción en una posición auxiliar netamente diferenciada de su posición definitiva, permiten a los constructores liberarse completamente del pesado y costoso inconveniente que constituye el empleo de las cimbras.

\section{9) Procedimientos para supmimir el empleo de cimbras}

Los procedimientos que vamos a describir consisten, esencialmente, en la ejecución de arcos por rebatimiento después del fraguado normal del hormigón de elementos constituyentes de un semiarco $(*)$ construido en posición vertical sobre los arranques formando articulaciones.

Así, pues, los procedimientos en cuestión suprimen completamente el empleo de cimbras y los inconvenientes que de ellas se derivan.

Además, por el hecho de hormigonar en posición vertical (construcción en castilletes) de los elementos precitados, los procedimientos mencionados permiten la realización de mejoras importantes en la construcción de los arcos y, principalmente:

por la posibilidad de empleo práctico y económico de encofrados metálicos deslizantes a lo largo de las partes de arco ya construidas, sobre las que se apoyan;

por la facilidad de colocación de las armaduras;

por la ejecución cómoda del hormigonado a lo largo de la sección del arco y por capas normales a la curva de presiones y, particularmente, por la gran facilidad de ejecución de los arcos tipo cajón (**).

En fin, el hecho de que los elementos de arco se hayan hormigonado en posición vertical y mantenidos libremente en esta posición hasta el fraguado normal del hormigón, suprimen en gran parte los esfuerzos debidos a la retracción del hormigón.

Cualquiera que sea el tipo y la luz, los arcos se realizan en principio bajo forma de arcos de triple articulación $\mathrm{y}$, si el caso lo requiere, pueden transformarse después, ya en arcos de doble articulación, ya en arcos empotrados. En estos dos casos, su construcción previa bajo forma de arcos de triple articulación ofrece la ventaja de fijar, con exactitud, los puntos principales de paso de la curva de presiones.

\section{Aplicación de los nuevos procedimientos}

Los procedimientos nuevos suprimen el empleo de cimbras y se aplican sin dificultades especiales a la construcción de diversos tipos de arcos de puente, principalmente:

puentes de arcos rebajados de pequeña, media y gran luz;

puentes de arcos peraltados (viaductos);

(*) Semiarcos en el caso general. Cuarta parte de arcos en las obras de grandes luces.

(**) Ver la figura adjunta. En el caso de arcos tipo cajón el procedimiento permite una ejecución particularmente cómoda del arco sobre toda su sección. 
obras destinadas a salvar, con gran altura, estrechos marítimos (*), estuarios de grandes ríos y valles amplios y profundos;

puentes con arcos atirantados;

puentes con tableros suspendidos;

puentes cantilever y bow-string.

5

Primer procedimiento ${ }^{* *}$

Construcción por semiarcos aislados

De acuerdo con el primer procedimiento, aplicable particularmente al caso de obras rebajadas de pequeñas luces, los arcos se realizan en dos semiarcos construidos aisladamente, en posición vertical, y sobre arranques, formando articulaciones (véase figura adjunta).

Después del fraguado normal del hormigón, estos semiarcos se rebaten hasta llevarlos a su posición definitiva (fig. 2) con ayuda de un cable fijado en su extremidad superior. Cada uno de estos semiarcos lleva un tirante provisional, que se tesa con objeto de oponerse a un aumento de luz hasta que se consiga la realización de la articulación central en la clave del arco completo (fig. 3$)(* * *)$.

\section{Segundo procedimiento $\left({ }^{* * *}\right)$}

Construcción con elementos de arco incorporados

con paneles indeformables, durante el rebatimiento

Una cimbra, por pequeña que sea su importancia, deberá ser estudiada y ejecutada con tanto cuidado como la propia obra. Algunas veces resulta más difícil su proyecto y construcción que la misma obra.

J. R. ROBINSON

\section{Primer caso: Amcos rebajados de Iuz media}

El segundo procedimiento consiste también en construir cada semiarco en posición vertical por encima de la zona de arranques correspondientes: pero en este caso incorporándola en una especie de panel destinado a oponerse totalmente a su deformación durante el curso del rebatimiento. Dicho panel se compone, por una parte, de elementos definitivos, de hormigón, que se colocaron al mismo tiempo que el del semiarco (montantes verticales u oblicuos y parte de la viga longitudinal) y, por otra parte, de elementos provisionales: tirante, como en el primer procedimiento, así como una o varias tornapuntas.

Siempre, y después del fraguado normal del hormigón, cada panel se rebate por medio de un cable fijado en la extremidad superior del semiarco, cuyo tirante fue previamente tesado para oponerse a un aumento de luz hasta la realización de la articulación en la clave.

(*) Véase «Génie Civil» del 16 de mayo de 1963, y Boletín mensual «S.A.D.G.» de la Asociación de Arquitectos D.T.L.G., núm. 118, junio 1963.

(**) Patente S.G.D.G. 1.234.757.

(***) Nótese la ejecución realizada en 1955 en Africa del Sur siguiendo un procedimiento sensiblemente diferente en un puente en arco, de hormigón armado y de $100 \mathrm{~m}$ de luz: dos semiarcos hormigonados en posición vertical sobre apoyos provisionales y rebatidos después con ayuda de un sistema de cables fijados en 4 puntos de cada uno de los semiarcos; éstos, dotados previamente de un dispositivo provisional de pretensado (36 cables desistencia colocados en el ex rante el rebatimiento y cuyo objeto es el de oponerse al cierre de la luz durante esta maniobra. Este procedimiento, casi totalmente desconocido en Europa, no parece haber experimentado otras aplicaciones.

(****) Patente S.G.D.G. 1.280.120. 
Por las disposiciones precedentes, que se oponen a toda formación del semiarco en el curso de su rebatimiento $\mathrm{y}$, por consiguiente, a la aparición de todo esfuerzo peligroso de tracción, el segundo procedimiento presenta la ventaja de ser aplicable, no solamente a la construcción de arcos de hormigón armado, sino también a la de arcos de hormigón no armado o, in extremis, débilmente armados. De todo ello resulta la posibilidad, para los constructores, de realizar una importante economía con la supresión de armaduras (siempre muy costosas).

Como precedentemente, los arcos se realizan previamente bajo forma de arcos de triple articulación. También puede, dado el caso, transformarse en arcos de doble articulación o en arcos empotrados.

\section{Aplicación del segundo procediumiento al Ia construcción de un puente con areo mebajado de mediana luz}

Las diversas fases de la construcción de tal obra pueden representarse esquemáticamente como sigue:

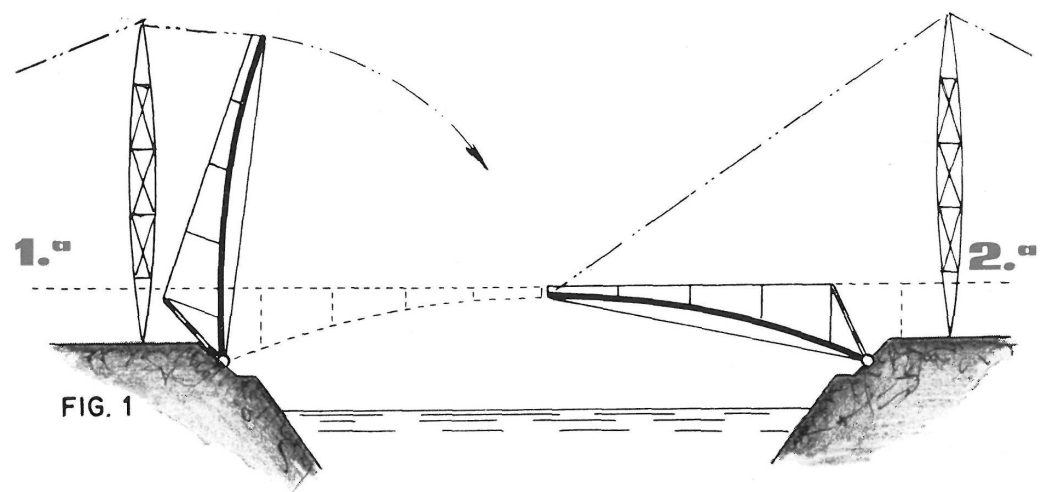

1. ‘ Fase:

A la izquierda de la fig. 1: Construcción de paneles hormigonados arranques formando articulaciones. $\mathrm{La}$ indeformabilidad de estos paneles se asegura con ayuda, por
una parte, de una tornapunta provisional (que aparece con un doble trazo y elleno discontinuo) $y$, por otra parte, de un tirante tam.

2.a Fase:

A la derecha de la fig. 1: Rebatimiento de los dos paneles por medio de un cable fijado en la extremidad superior de cada semiarco, operación seguida de la colocación ostramiento.

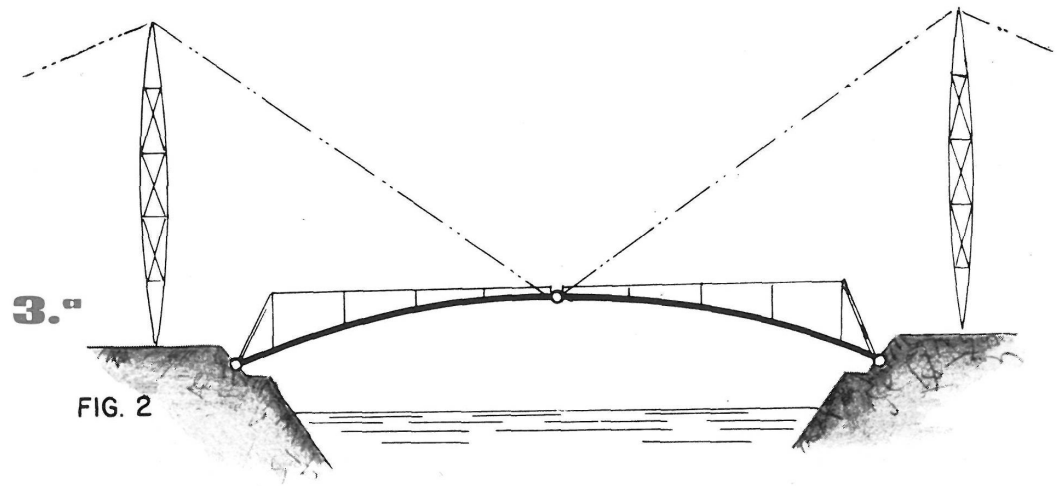

\section{3. ${ }^{\circ}$ Fase:}

Fig. 2: Realización de la articuación central, seguida de la sules $y$ tirantes.

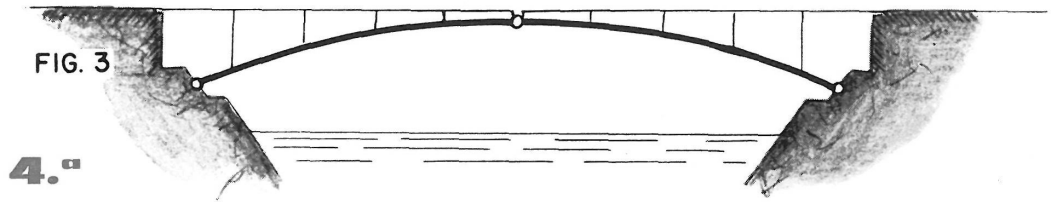

\section{4. ${ }^{\circ}$ Fase:}

Fig. 3: Terminación de la obra. 


\section{Aplicución del segundo procedimiento de la consturectón

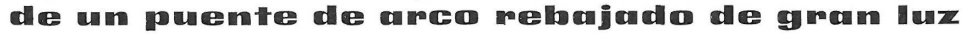

En un arco de gran luz, el factor más importante no es el arco, sino la cimbra, y su consideración tiene interés preeminente en la elección de las disposiciones del arco.

J. R. ROBINSON

- los arcos rebajados de grandes luces se construyen por medio de cuatro paneles indeformables (cuarta parte del arco), análogos a los precedentes y simétricos dos a dos;

- dos paneles laterales, los más importantes, construidos directamente sobre los arranques (véanse las figuras);

- dos paneles centrales, construidos después de rebatir los paneles laterales sobre la extremidad superior del elemento de arco formando articulación intermedia provisional.

La parte izquierda de las figuras muestra un primer modo operatorio, sin apoyo intermedio, correspondiente al caso de un tráfico que exige entera libertad de paso bajo la obra en construcción. La parte derecha de las mismas figuras se refiere a un segundo modo operatorio que necesita la utilización de un apoyo intermedio al aplomo de la extremidad superior de cada uno de los dos paneles laterales, lo que permite mayor facilidad de construcción y deja todavía un amplio espacio para el tráfico.

\section{1.a Fase:}

A la izquierda de la fig. 4: Cons. trucción de paneles laterales cololos arranques. Como precedentemente, la indeformabilidad de es. tos paneles se realiza con ayuda de dos tornapuntas provisionales trazo doble y relleno intermitenprovisional (trazo discontinuo)

2. Fase:

A la derecha de la fig. 4: Rebay colocación de un dispositivo transversal en estos paneles.

\section{3. a Fase:} A la izquierda de la fig. 5: Cons.
trucción en posición vertical de
los paneles del centro sobre articulos paneles del centro sobre articudas por un pequeño círculo) entaextremidad superior de cada uno de los paneles laterales.

\section{4." Fase}

A la derecha de la fig. 5: Rebatimiento de los paneles centrales, seguido de su arriostramiento trans versal y de la rigidización de las articulaciones intermedias entre pa neles centrales $\mathbf{y}$ laterales. Reali(clave).

\section{5. a Fase:}

Fig. 6: El conjunto constituye un arco de tres articulaciones con su. presión de tirantes, tornapuntas $y$
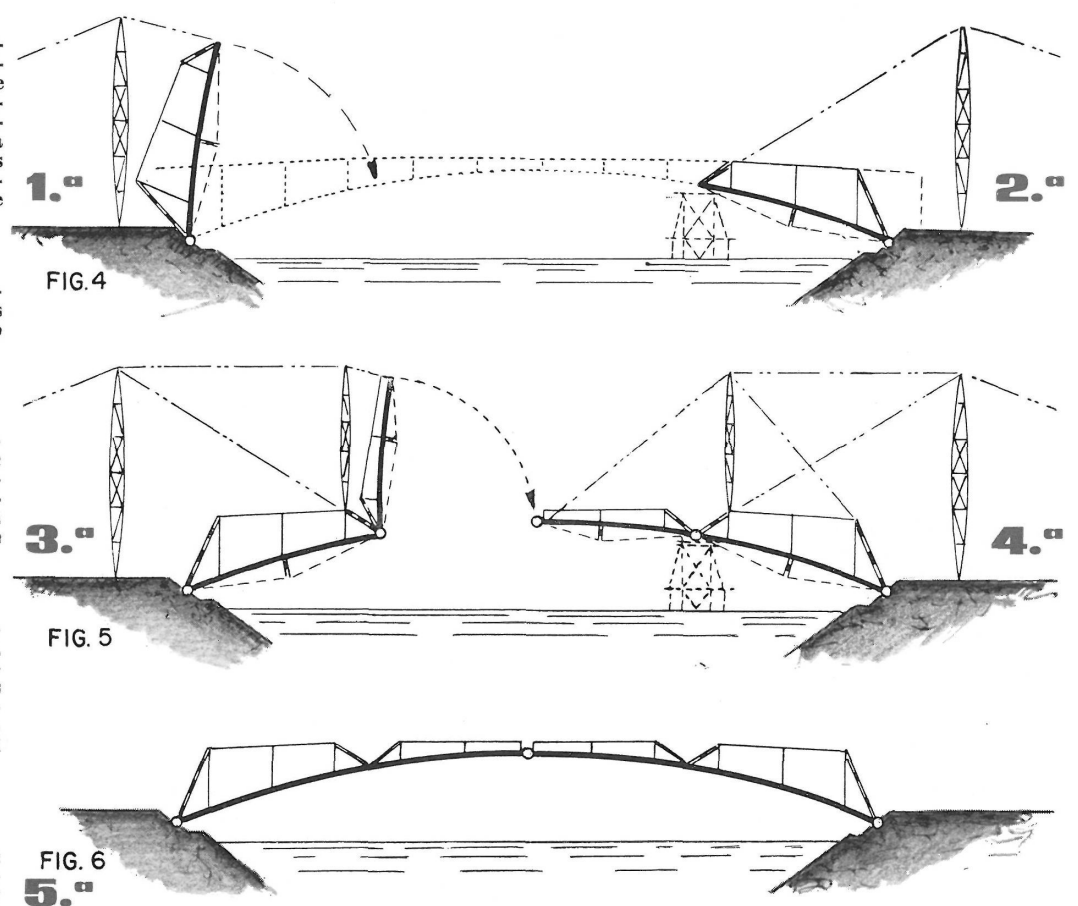


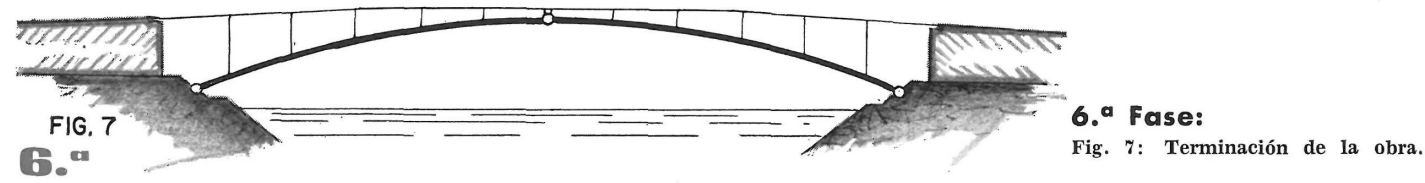

\section{Aplicución del segundo procedimiento a lea construcción de puentes con arcos peraltados [viaductos]}

La construcción, siguiendo los procedimientos clásicos, de obras con arcos peraltados, necesita del empleo de cimbras particularmente importantes y, cuya ejecución, frecuentemente difícil, es siempre extremadamente costosa.

También, el segundo procedimiento tiene una aplicación particularmente interesante en la construcción de estas obras.

Como los arcos rebajados de gran luz, los arcos peraltados se ejecutan por medio de cuatro elementos de arco simétricos dos a dos.

- dos elementos de base: simples porciones de arcos que pueden ser ejecutadas con hormigón no armado o en su caso débilmente armado;

- dos elementos centrales: paneles indeformables análogos a los paneles centrales de las obras rebajadas de gran luz.

Las diversas fases de la construcción de arcos peraltados pueden representarse esquemáticamente:

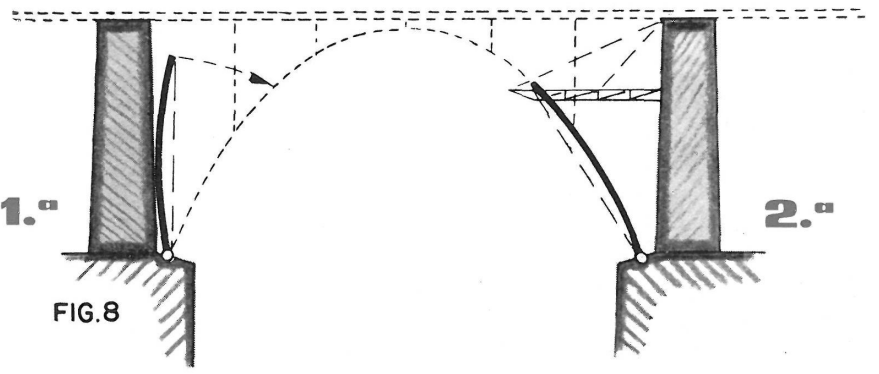

1. ${ }^{a}$ Fase:

A la izquierda de la fig. 8: Construcción de los elementos de base, hormigonados verticalmente te con tirantes tesados provisionalmente (trazo discontinuo).

2. a Fase:

A la derecha de la fig. 8: Rebatimiento de los elementos de base hasta sus posiciones respectivas en las que se mantienen por medios conveas laterales.

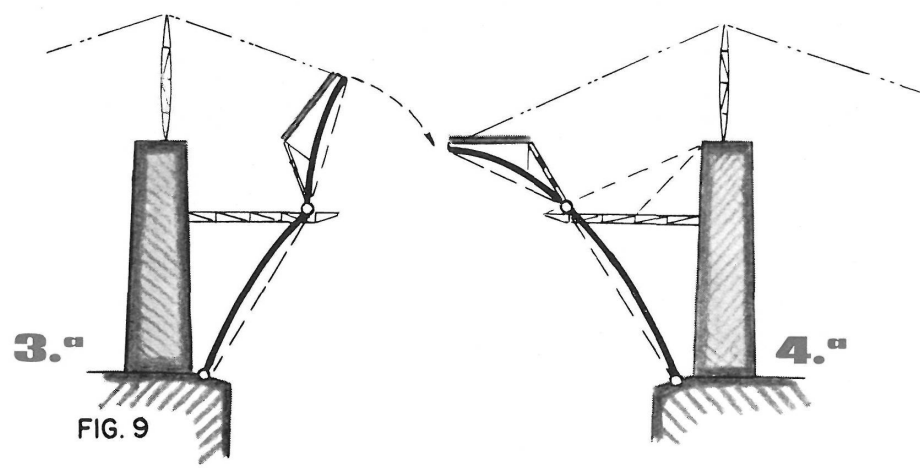

3. ' Fase:

A la izquierda de la fig. 9: Construcción de los paneles centrales, hormigonados en posicion verticulación provisional intermedia de cada elemento de base.

\section{4." Fase:}

A la derecha de la fig. 9: Rebatimiento de paneles centrales, seguido de la ejecución parcial 

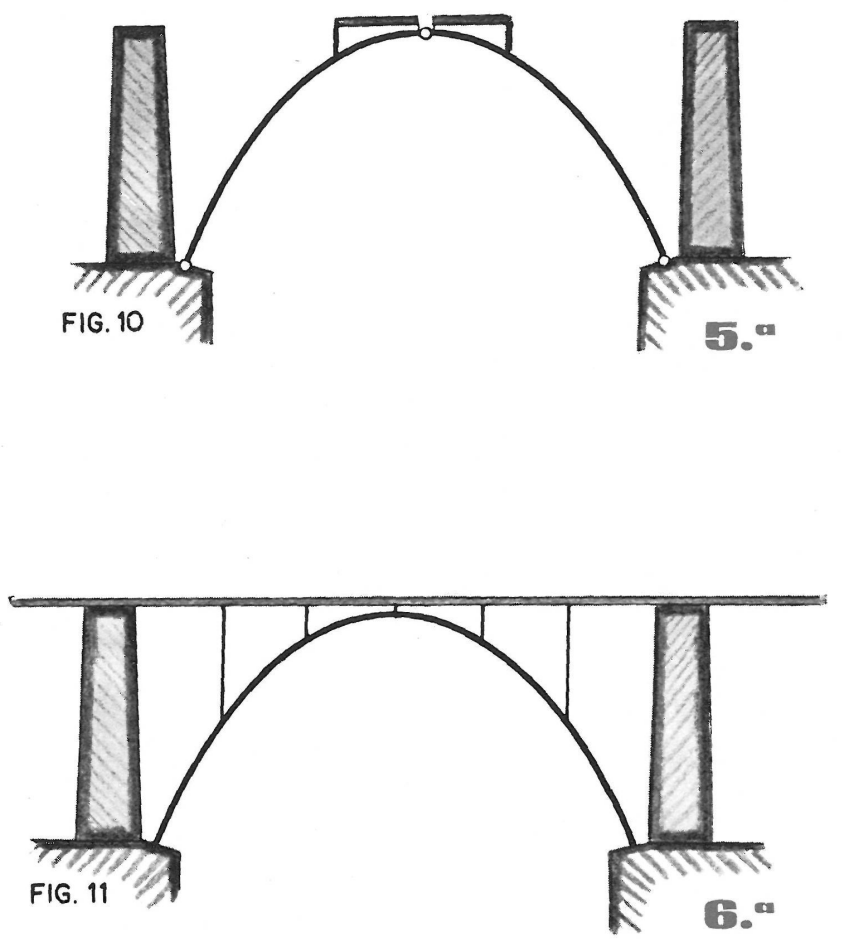

\section{Fase:}

Fig. 10: Rigidización de las articulaciones pro-
visionales intermedias, seguida de la articulación visionales intermedias, seguida de la articulación
central y de la supresión de tornapuntas, tirantes y pasarelas. EI conjunto de la construcción constituye un arco de tres articulaciones.

\section{Fase:}

Fig. 11: Transformación del arco de tres articu. laciones en arco empotrado por rigidización de

\section{Aplicación del segumdo procedimiemto: \\ Construcción de arcos contiguos de gran luz descansando sobre pilas de gran altura (Paso sobre estrechos marítimos y estuarios de grandes ríos)}

Una cuarta aplicación del segundo procedimiento se refiere a la construcción de obras de importancia excepcional, destinadas a salvar con grandes alturas estrechos marítimos y grandes estuarios, obras constituidas por una o varias series de grandes arcos contiguos de hormigón armado (*) (véase la revista «Le Génie Civil», 15 de mayo de $1963(* *)$, pág. 216, «Aplicación del procedimiento a la construcción de arcos contiguos para el paso del estrecho de Calais»).

La construcción de estos arcos contiguos (arcos atirantados de $200 \mathrm{~m}$ de luz útil), que deben realizarse sobre el mar entre las cotas $+58 \mathrm{a}+82 \mathrm{y}$, por tanto, comportar eventualmente un doble o triple tablero, se efectuaría simétricamente (por semiarcos) respecto a cada una de las pilas:

Traducido y adaptado por $J . J$. Ugarte.

(*) Nótese que (la simple cuestión de cimbras) tales obras son prácticamente irrealizables con los procedimientos clásicos.

(**) Bajo el título "Procedimiento para la construcción sin cimbra, de un puente en arco, de hormigón armado, de doble o triple tablero, para el paso sobre el Paso de Calais. 


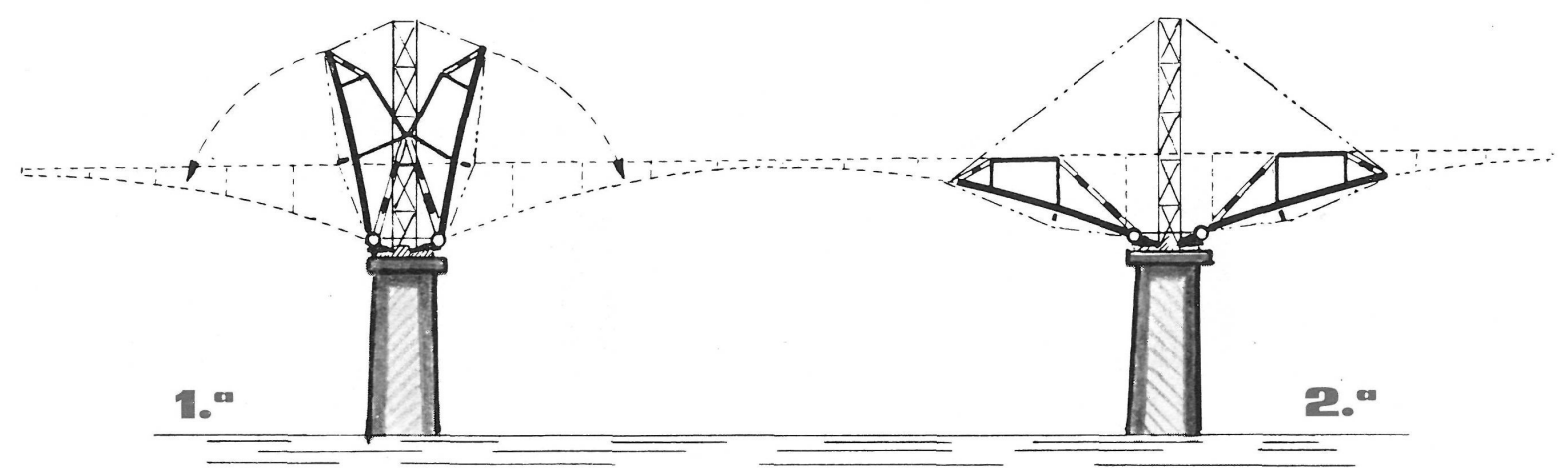

FIG. 12

1. a Fase: A la izquierda de la fig. 12: Construcción simultánea (sobre extremidades de arcos $\left({ }^{*}\right)$ de los paneles laterales.

2. Fase: A la derecha de la fig. 12: Rebatimiento simultáneo de los paneles laterales.

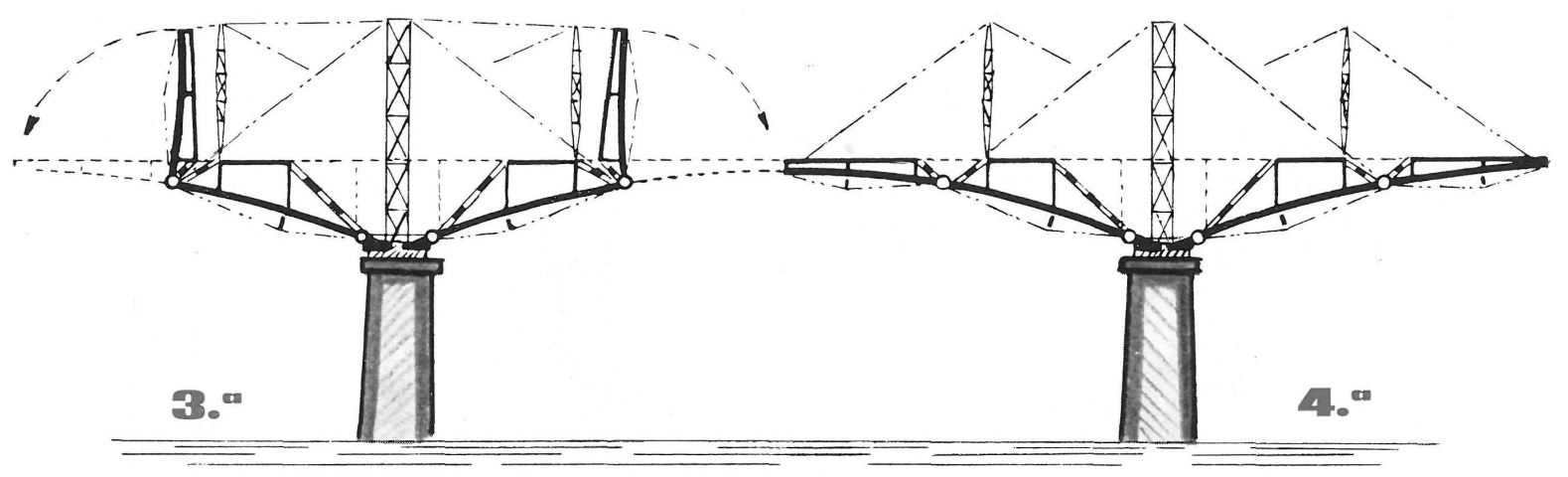

FIG. 13

3. a Fase: A la izquierda de la fig. 13: Construcción simultánea de los paneles centrales en las extremidades, formando articulaciones provisionales intermedias de los paneles laterales.

4. a Fase: A la derecha de la fig. 13: Rebatimiento simultáneo de los paneles laterales.

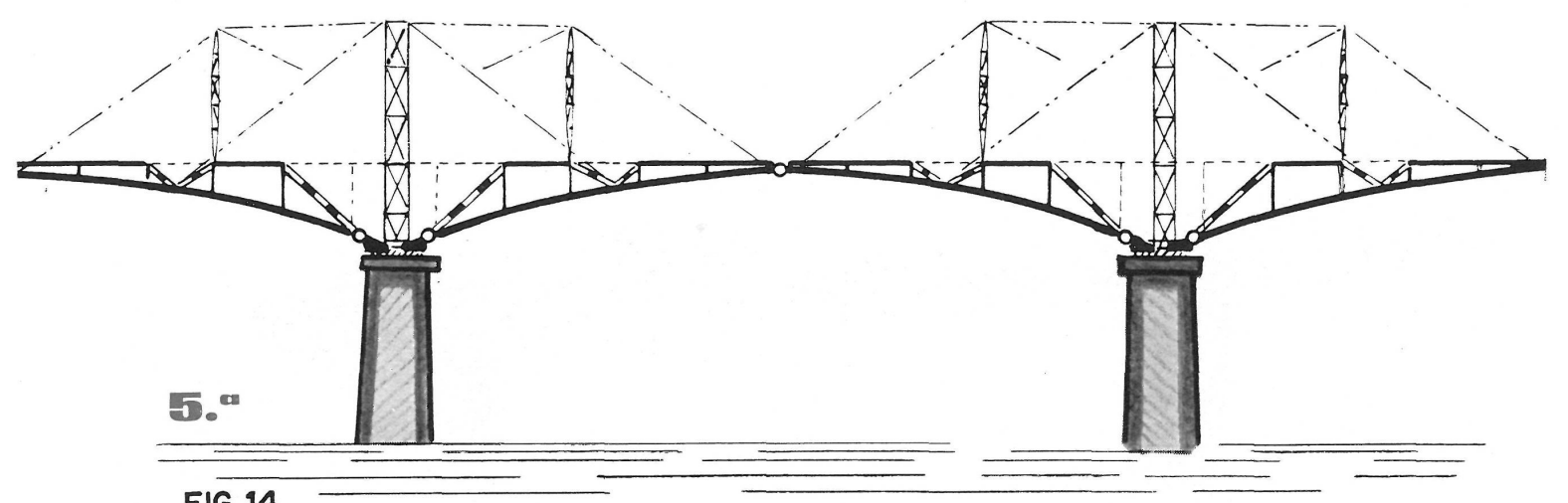

FIG. 14 5. ‘ Fase: Fig. 14: Rigidización de las articulaciones intermedias (entre paneles laterales y paneles centrales). Realización de la articu-
lación central (clave).

(*) Sobre la construcción de estas extremidades en arco, consúltese el artículo precitado del «Génie Civil», pág. 216. 


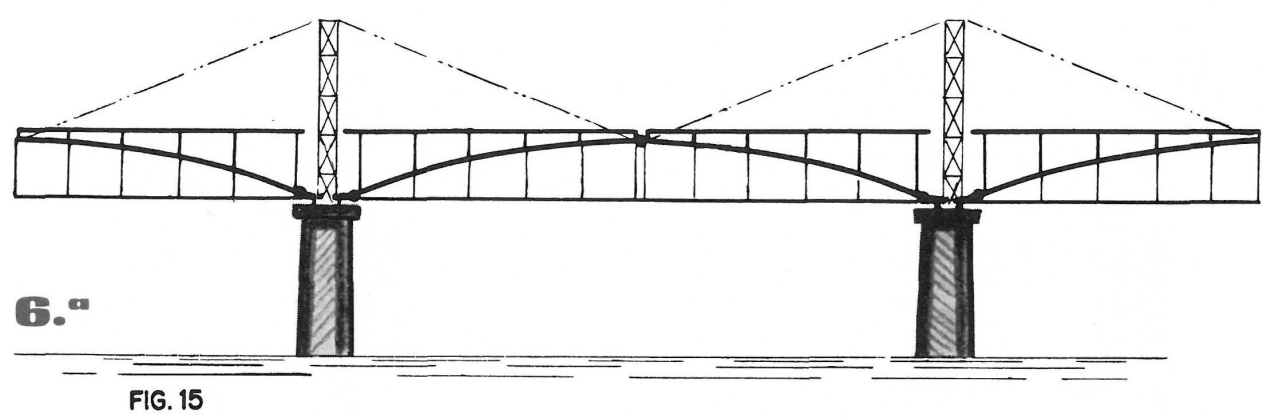

FIG. 15

\section{6." Fase:}

Fig. 15: Colocación de la suspensión y tirantes. Levantamiento de los montantes verticales $\mathbf{y}$

de las vigas longitudinales.

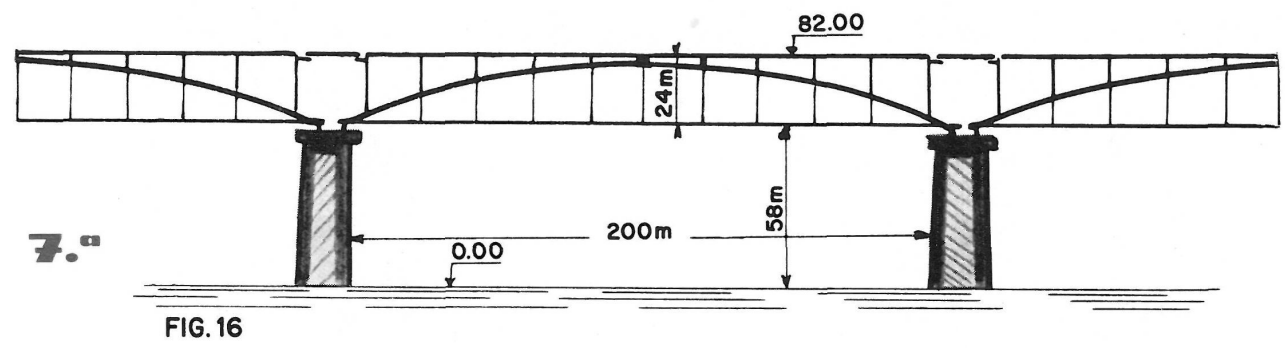

7.a Fase:

Fig. 16: Supresión

de las tornapuntas,

cables de retención y tirantes provisionales. Terminación

de la obra.

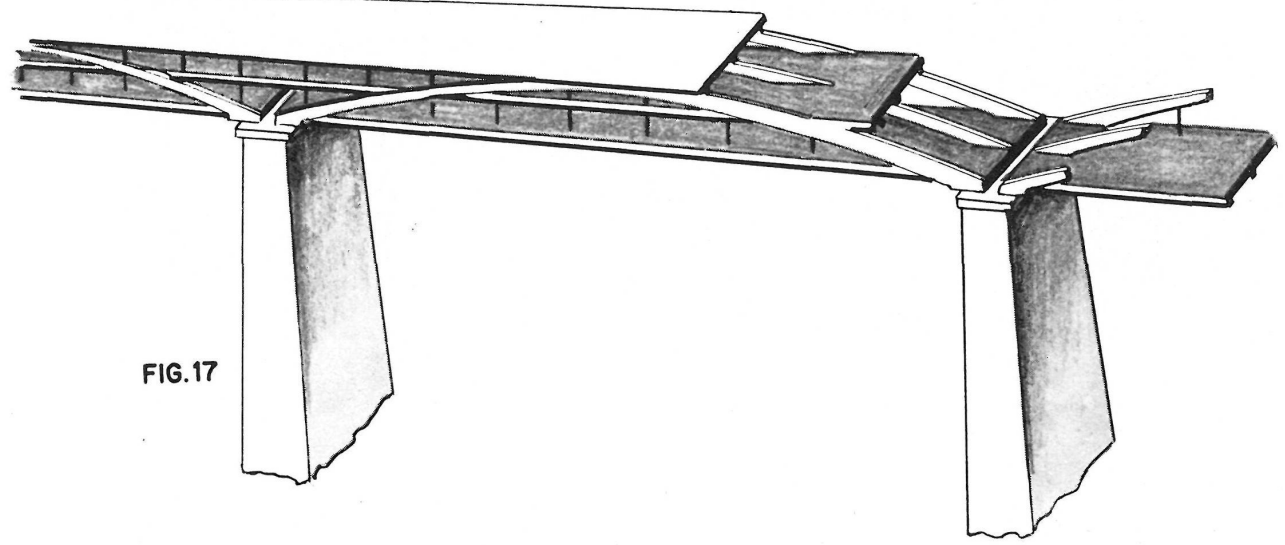

Fig. 17: Perspectiva de un tramo con triple tablero. 


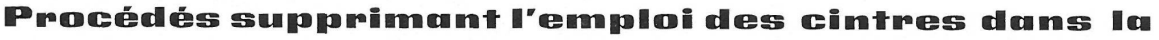

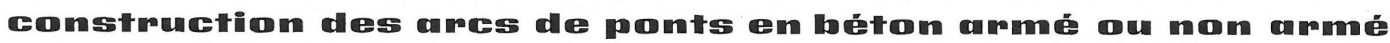

Paul Dupont, ingénieur

Dans ce travail est décrit en détail les différents moyens et procédés qui peuvent être employés pour supprimer le cintre auxiliaire dans la construction d'arcs et de voûtes des ponts en béton armé ou non armé.

L'emploi du cintre, lourd et coûteux, pourrait être supprimé non seulement pour les portées de faible importance ayant des appuis en terre ferme, mais aussi pour les arches de grande portée et même au-dessus de la mer, comme pour franchir les estuaires des grands fleuves, ou des détroits, comme c'est le cas, par exemple, pour le Pas-de-Calais.

\section{Procedure for avoiding the use of scaffolding in meinforced or unmeinforced concrete bridges}

Paul Dupont, engineer

This paper gives a detailed description of the various procedures that may be used to avoid the need of scaffolding in the construction of arches and bridge vaults, made either of reinforced or unreinforced concrete.

The scaffolding is expensive and cumbersome, and could be eliminated not only in the case of small spans on firm ground, but also in the case of large spans over seawater, $i$. e., over estuaries of large rivers, or narrows; for instance in the case of the «Pas de Calais».

\section{Verfolhmung fiin alie Bescitigung dem Amwendung won

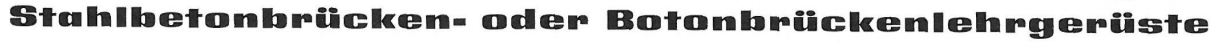

Paul Dupont, Ingenieur

In dieser Arbeit wurden weitgehend die verschiedenen Mittel und Verfahrungen beschrieben, die angewendet werden köonnen, um die Anwesenheit des Hilfslehrgerüstes im Bogen- und Gewölbebau von Stahlbeton- oder Betonbrücken abzuschaffen. Das Lehrgerüst, teuer und lästig, könnte vermeidet werden, nicht nur in kleinen Spannweiten von kleiner Wichtigkeit mit Stütze auf festen Boden, sondern auch in grosser Spannweite und über dem Meer, das ist, über enger Flussmündung von grossen Flüsse, Fall, in der sich der «Pas-de-Calais» findet. 